\title{
Editorial:
}

\section{Progress and challenges in the cardiovascular field}

\author{
Jian-an WANG ${ }^{1,2}$ \\ Guest Chief Editor, \\ Chairman of Qianjiang International Cardiovascular Congress \\ $\left({ }^{I}\right.$ Department of Cardiology, the Second Affiliated Hospital, School of \\ Medicine, Zhejiang University, Hangzhou 310009, China) \\ $\left({ }^{2}\right.$ Cardiovascular Key Lab of Zhejiang Province, the Second Affiliated \\ Hospital, School of Medicine, Zhejiang University, Hangzhou 310009, \\ China) \\ E-mail: Wang_jian_an@tom.com
}

doi:10.1631/jzus.B1201010

Despite great achievements in their treatment and prevention in the past few decades, cardiovascular diseases remain the leading cause of human mortality and morbidity all around the world. As estimated by the world health organization (WHO) in 2008, over 17 million people die from cardiovascular diseases every year. It is reported that in China we have around 23 million cardiovascular disease patients, along with a significant disease burden (Zhao and Liu, 2012). Business corporations and non-profit health organizations, physicians and biomedical scientists are making great progress in preventing and treating cardiovascular diseases. Due to the globalization of information technology, we are able to access the latest progress in the biomedical field, or look back to the achievements of past decades. However, there are still some ways to go before our guidelines or expert consensus can be used to benefit patients (Huo, 2010). Moreover, numerous new diagnostic techniques and therapeutic methods (including drugs, devices, biomedical materials, and surgical operations) are created every year, making it more and more difficult for physicians to make the optimal choice for patients (Wang, 2010). Therefore, it is of vital importance to introduce, interpret, discuss and summarize information on cardiovascular diseases.

Qianjiang International Cardiovascular Congress (QICC) is aiming to be the bridge and catalyst between patients and physicians, amateurs and experts, biomedical scientists and clinical physicians, as

(C) Zhejiang University and Springer-Verlag Berlin Heidelberg 2012 described in its theme: focus on prevention, comply with guidelines, start from the beginning, go to the summit. Previously we've successfully organized 5 QICC scientific sessions. The 6th QICC (QICC6) and 2012 Scientific Session of the Zhejiang Society of Cardiology will be held in Hangzhou from Aug. 2 to Aug. 5, 2012. It is our great honor and pleasure to have the Agioplasty Summit of Transcatheter Cardiovascular Therapeutics Asia Pacific (TCTAP), Korea and the National University Heart Center, Singapore (NUHCS) as our new co-sponsors this year. Both the Taiwan Society of Cardiovascular Interventions (TSCI), and the Hong Kong College of Cardiology (HKCC) will also support QICC6 as usual. Outstanding doctors and scientists worldwide will join this academic conference by giving lectures, demonstrating procedures, and commenting on speeches and posters to share their knowledge and experience.

Since 2010, the Journal of Zhejiang UniversitySCIENCE B (Biomedicine \& Biotechnology) has published two special issues for the QICC conference, which have remarkably helped the delivery of new concepts and expertise in the cardiovascular field. Ischemic heart disease, especially coronary heart disease (CHD), is the leading cause of cardiovascular death, and percutaneous coronary intervention (PCI) has become the most common procedure for treating CHD. However, there are still several challenges for PCI techniques. Re-canalization of chronic total occlusion (CTO) requires extraordinary techniques and innovative devices, and is considered to be at the cutting edge of PCI (Kim et al., 2010; Li et al., 2010). In this special issue, we are delighted to have Dr. Jun-bo GE introduce the current status of PCI in relation to CTO (Ge, 2012). Other PCI techniques, such as rotational atherectomy for complex coronary lesions, are also included in this issue (Jiang et al., 2012).

Atrial fibrillation (AF) is a very common arrhythmia in clinical practice, with an increasing trend in the elder population (Lip et al., 2012). It has been well demonstrated that $\mathrm{AF}$ is a strong independent risk factor for stroke (Lip, 2011). Current guidelines recommend long-term anticoagulation therapy for AF patients. However, the complexity of monitoring the 
international normalized ratio (INR) in AF patients taking warfarin affects compliance and sometimes causes fatal hemorrhage. A review paper by Dr. Shu ZHANG focusing on novel anticoagulation agents and the adjustment of anticoagulation therapy in certain situations is highlighted in this issue (Zhang, 2012).

The role of variable RNA splicing in the transcriptome has evoked great interest among scientists in recent years (Nanni et al., 2006; Zeller et al., 2012). Different RNA splicing variants could eventually turn out to be protein products with distinctly different functions. Dr. Yibin WANG from the University of California at Los Angeles (UCLA) will discuss the current progress in alternative RNA splicing in cardiac remodeling, introduce the latest technology, and discuss the global impact of RNA splicing in developmental and pathological situations (Gao and Wang, 2012).

Bone marrow-derived mesenchymal stem cell (MSC) transplantation has been demonstrated to be a promising alternative method for attenuating adverse cardiac remodeling and restoring heart function after myocardial infarction. Cell survival rate in the harsh environment is the key factor affecting the therapeutic effect of stem cell transplantation (Gao et al., 2010; Hu et al., 2011). In this special issue, pharmacological preconditioning of MSCs with angiopoitetin-1 is shown to further improve cardiac function through a pro-survival mechanism, suggesting a possibility of preconditioning MSCs without genetic manipulation to enhance their therapeutic effect (Liu et al., 2012).

Of note, we will have the 30th anniversary of the Journal of Electrocardiology (China) in the 6th QICC scientific sessions. Founded by Dr. Yang ZHAO in 1982, the Journal of Electrocardiology has contributed greatly to the establishment and development of electrocardiology in China. To incorporate the explosive progress in the cardiovascular field, this journal has since changed its name to the Journal of Electrocardiology and Circulation as of January 2012. We are dedicated to upholding the reputation of this journal in bringing the latest progress in the cardiovascular field.

On behalf of the organizing committee of QICC6 and the Zhejiang Society of Cardiology, we sincerely welcome your participation in this conference. We hope QICC6 will be a bridge and catalyst for sharing our knowledge, experience, passion and love for treating cardiovascular diseases.

\section{References}

Gao, C., Wang, Y., 2012. Global impact of RNA splicing on transcriptome remodeling in the heart. J. Zhejiang Univ.-Sci. B (Biomed. \& Biotechnol.), 13(8):603-608.
[doi:10.1631/jzus.B1201006]

Gao, F., Hu, X.Y., Xie, X.J., Xu, Q.Y., Wang, Y.P., Liu, X.B., Xiang, M.X., Sun, Y., Wang, J.A., 2010. Heat shock protein 90 protects rat mesenchymal stem cells against hypoxia and serum deprivation-induced apoptosis via the PI3K/Akt and ERK1/2 pathways. J. Zhejiang Univ.-Sci. B (Biomed. \& Biotechnol.), 11(8):608-617. [doi:10.1631/ jzus.B1001007]

Ge, J.B., 2012. Current status of percutaneous coronary intervention of chronic total occlusion. J. Zhejiang Univ.-Sci. B (Biomed. \& Biotechnol.), 13(8):589-602. [doi:10.1631/jzus.B1201009]

Hu, X.Y., Wang, W.X., Yu, M.J., Liu, X.B., Wu, R.R., Gao, F., Huang, X., Cao, J., Xie, X.J., Wang, J.A., 2011. Tongxinluo promotes mesenchymal stem cell tube formation in vitro. J. Zhejiang Univ.-Sci. B (Biomed. \& Biotechnol.), 12(8):644-651. [doi:10.1631/jzus.B1101005]

Huo, Y., 2010. Current status and development of percutaneous coronary intervention in China. J. Zhejiang Univ.-Sci. B (Biomed. \& Biotechnol.), 11(8):631-633. [doi:10.1631/jzus.B1001012]

Jiang, J., Sun, Y., Xiang, M.X., Dong, L., Liu, X.B., Hu, X.Y., Feng, Y., Wang, J.A., 2012. Complex coronary lesions and rotational atherectomy: one hospital's experience. $J$. Zhejiang Univ.-Sci. B (Biomed. \& Biotechnol.), 13(8): 645-651. [doi:10.1631/jzus.B1201008]

Kim, W.T., Ahn, S.G., Lee, J.W., Sung, J.K., Lee, S.H., Yoon, J., 2010. Successful recanalization of chronic total occlusion of the superior mesenteric artery by transradial approach. J. Zhejiang Univ.-Sci. B (Biomed. \& Biotechnol.), 11(8):627-630. [doi:10.1631/jzus.B1001014]

Li, P., Gai, L.Y., Yang, X., Sun, Z.J., Jin, Q.H., 2010. Computed tomography angiography-guided percutaneous coronary intervention in chronic total occlusion. $J$. Zhejiang Univ.-Sci. B (Biomed. \& Biotechnol.), 11(8): 568-574. [doi:10.1631/jzus.B1001013]

Lip, G.Y., 2011. Atrial fibrillation in 2011: stroke prevention in AF. Nat. Rev. Cardiol., 9(2):71-73. [doi:10.1038/ nrcardio.2011.203]

Lip, G.Y., Tse, H.F., Lane, D.A., 2012. Atrial fibrillation. Lancet, 379(9816):648-661. [doi:10.1016/S0140-6736(11) 61514-6]

Liu, X.B., Chen, H., Chen, H.Q., Zhu, M.F., Hu, X.Y., Wang, Y.P., Jiang, Z., Xu, Y.C., Xiang, M.X., Wang, J.A., 2012. Angiopoietin-1 preconditioning enhances survival and functional recovery of mesenchymal stem cell transplantation. J. Zhejiang Univ.-Sci. B (Biomed. \& Biotechnol.), 13(8):616-623. [doi:10.1631/jzus.B1201004]

Nanni, L., Romualdi, C., Maseri, A., Lanfranchi, G., 2006. Differential gene expression profiling in genetic and multifactorial cardiovascular diseases. J. Mol. Cell. Cardiol., 41(6):934-948. [doi:10.1016/j.yjmcc.2006.08.009]

Wang, J.A., 2010. Advances in cardiovascular disease: how far is the summit? J. Zhejiang Univ.-Sci. B (Biomed. \& Biotechnol.), 11(8):547. [doi:10.1631/jzus.B1001015]

Zeller, T., Blankenberg, S., Diemert, P., 2012. Genomewide association studies in cardiovascular disease-an update 2011. Clin. Chem., 58(1):92-103. [doi:10.1373/clinchem. 2011.170431]

Zhang, S., 2012. Clinical considerations of anticoagulation therapy for patients with atrial fibrillation. J. Zhejiang Univ.-Sci. B (Biomed. \& Biotechnol.), 13(8):609-615. [doi:10.1631/jzus.B1201007]

Zhao, D., Liu, J., 2012. Cardiovascular disease in China: increasing burden ahead for prevention. Chin. J. Cardiol., 40(3):177-178 (in Chinese). 